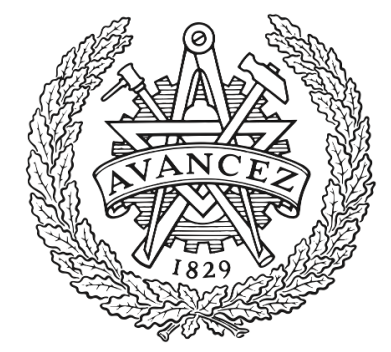

\title{
CHALMERS
}

UNIVERSITY OF TECHNOLOGY

\section{Power-Efficient ASIC Implementation of DSP Algorithms for Coherent Optical Communication}

Downloaded from: https://research.chalmers.se, 2023-04-26 14:06 UTC

Citation for the original published paper (version of record):

Larsson-Edefors, P., Börjeson, E. (2020). Power-Efficient ASIC Implementation of DSP Algorithms for Coherent Optical Communication. 2020 IEEE Photonics Society Summer Topical Meeting Series, SUM 2020 - Proceedings, July 2020. http://dx.doi.org/10.1109/SUM48678.2020.9161072

N.B. When citing this work, cite the original published paper. 


\title{
Power-Efficient ASIC Implementation of DSP Algorithms for Coherent Optical Communication
}

\author{
Per Larsson-Edefors and Erik Börjeson \\ Chalmers University of Technology, Gothenburg, Sweden \\ perla@chalmers.se
}

\begin{abstract}
Coherent optical communication critically relies on efficient digital signal processing (DSP). We outline the application-specific integrated circuit (ASIC) implementation flow for DSP algorithms and discuss approaches to reducing the digital ASIC power dissipation of high-throughput DSP implementations for coherent fiber-optic communication systems.
\end{abstract}

\section{Power-Aware ASiC Implementation: A Primer}

Digital ASIC design involves a number of phases, and electronic design automation (EDA) tools, as shown in Fig. 1. Once a fixedpoint model of an algorithm has been developed, the designer creates a hardware description language (HDL) implementation containing different digital modules. Since throughput requirements are very strict for fiber-optic communication systems, choice of parallelism and pipelining is essential: The bit rate can be increased either by parallelizing hardware in several data lanes or inserting synchronization elements that pipeline the computations, effectively increasing the clock rate. In algorithms with feedback, however, it is far from straightforward to apply these speed-enhancing techniques.

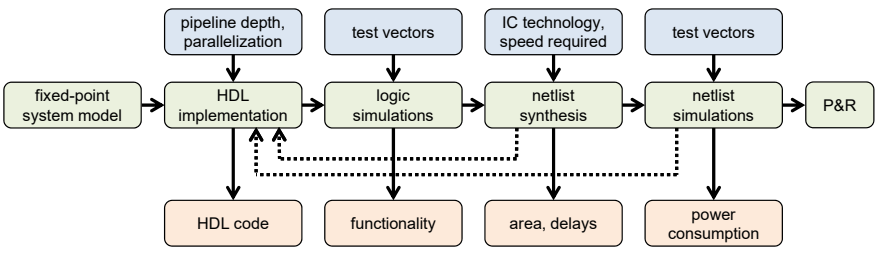

Fig. 1. Digital ASIC implementation phases.

Once the HDL code has been thoroughly verified for functionality using logic simulations, the next EDA tool performs netlist synthesis. With information on the IC technology and its logic gate cells, the synthesis tool maps the HDL code into a cell netlist under a timing constraint, which is the longest delay that we can accept between any two synchronizing elements, to sustain the required bit rate. If the timing constraint is not met, the designer needs to optimize the HDL implementation or, worse, reconsider the whole design approach.

In digital CMOS circuits, power dissipation is either static (leakage in off-state transistors) or dynamic (switching gate outputs). Leakage is mainly a problem in performance-oriented IC technologies, in which switching speed is prioritized. The always present switching power dissipation, $P_{s w}$, is caused by charges brought from the power supply down to ground to charge and discharge logic gate outputs. Assuming $Q$ is the charge and $V_{D D}$ is the supply voltage, an energy of $Q \cdot V_{D D}=\left(C V_{D D}\right) \cdot V_{D D}$ is dissipated during a charge-discharge cycle of a gate output with capacitance $C$. For a netlist with $N$ logic cells,

$$
P_{s w}=f V_{D D}^{2} \sum_{i=1}^{N}\left(C_{i} \alpha_{i}\right) .
$$

Here, $f$ is the clock rate, while $C_{i}$ and $\alpha_{i}$ is the capacitance and switching activity, defined as the fraction of cycles when a gate output switches from 0 to 1 , respectively, of the output of gate $i$. To identify $\alpha_{i}$, we perform netlist simulations based on meaningful test vectors.
The final phase needed to complete the ASIC design is to run place and route $(P \& R)[1]$, in which logic cells are placed by abutment, inter-cell logic signal wires routed, and a clock network is constructed. This completes the physical implementation and provides very accurate layout-based data from which to estimate area usage, longest delay, and power dissipation. It should be noted, however, that modern synthesis tools for digital ASICs can often accurately estimate wire lengths without having to perform full P\&R.

\section{DESIGN OF FEC CIRCUITS}

While DSP often has come to include forward error correction (FEC), these two functions have little in common. In fact, DSP circuits are mainly performing filtering to continuously shape the data stream of a signal, while the FEC circuit is, for most of the time, only monitoring a stream of digital data. The actual correction of an error does cause significant power dissipation, but this event is rare [2]. In fiber-optic communication, the FEC module is often specified to reduce a pre-FEC bit-error rate (BER) around $10^{-3}-10^{-2}$ to a postFEC BER of $10^{-15}$. This means the switching power dissipation is higher in early iterations, at the front-end of the FEC module [3].

Since observable post-FEC errors are extremely rare, the analysis of coding gain and error floor is recognized to be a formidable challenge: One of our field-programmable gate array (FPGA) prototypes was running continuously for 30 days to capture statistically stable data for a post-FEC BER of $10^{-15}$ [4] and in a post-deadline paper at OFC'18, ZTE demonstrated a system with 50 FPGAs to explore deep-BER behavior of FEC circuits intended for OIF 400G ZR [5].

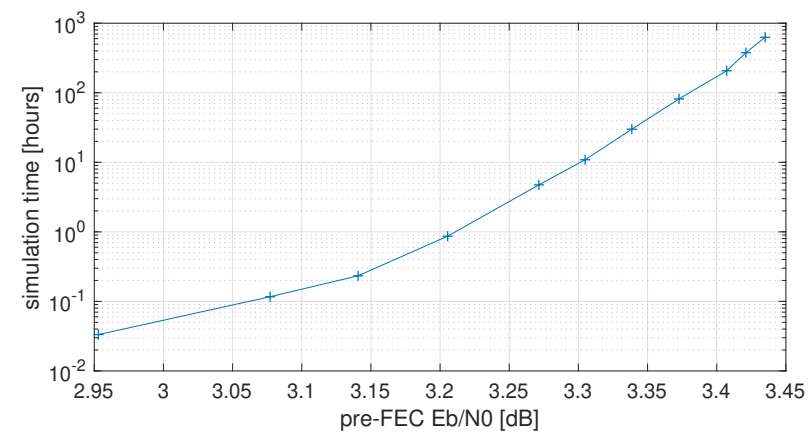

Fig. 2. Simulation time of decoder HDL code as function of $E_{\mathrm{b}} / N_{0}$.

Since FEC power dissipation depends strongly on how many errors are corrected, netlist simulations (Fig. 1) are instrumental to an accurate power analysis. But, again, since the correction of an error is rare, FEC simulations in general are very time consuming. Fig. 2 shows the time it takes to perform logic simulations (Fig. 1) for a BER analysis of a product decoder [3] based on $\mathrm{BCH}(115,94,3)$ component codes. The longest simulation corresponds to a run-time of 26 days, yet this reaches only down to a post-FEC BER of $3 \cdot 10^{-8}$.

Since they only occasionally correct erroneous data in the stream, FEC circuits can be aggressively optimized for low power dissipation. 
For example, we have demonstrated hard- and soft-decision FEC circuits for optical communication, with bit rates in the range of $400 \mathrm{~Gb} / \mathrm{s}$ to $1 \mathrm{~Tb} / \mathrm{s}$, having energy efficiencies of around $1 \mathrm{pJ} / \mathrm{bit}$ [3], [4], [6]. We prefer a metric of energy efficiency, i.e., energy per bit,

$$
E_{\text {bit }}=P_{\text {sw }} / \text { throughput, }
$$

over absolute power dissipation as it allows us to conveniently perform design tradeoffs between different system components.

\section{DESIGN OF DSP CIRCUITS}

Since coherent schemes require sophisticated DSP functions, DSP power dissipation is an issue [7]. In contrast to FEC, DSP circuits operate on each data sample and thus have high switching activities. This severely restricts the available power-saving design options.

A good starting point for ASIC design can be to separate the part of the DSP algorithm that is involved in estimation of signal properties from the actual operation on the data samples. The fundamental reason for this is that the estimation circuits may not need the full parallelism required by operations at full bit rate: For the adaptive equalizer, which we expect to dominate DSP power dissipation for 20-100-km coherent links [8], we can reduce the power dissipation by more than $50 \%$ via simplifications to the tap update algorithm [9].

To save power in DSP circuits, simplified coherent receiver architectures can be used [10]. A self-homodyne receiver represents a lower limit of DSP functions: One polarization is sacrificed for a pilot tone, to avoid the local oscillator [11]. But for the sake of throughput, we would prefer to use the full optical field. If we scale a conventional coherent receiver architecture down to shorter reaches, we can neglect some impairments, e.g., chromatic and polarization-mode dispersion, which allows us to use only a short adaptive equalizer. This in turn will move the design focus to analog-digital converters (ADCs) [12] and DSP components that are indispensable for any fiber length.

Unless pilot symbols are used, the carrier phase recovery (CPR) module will constitute a significant portion of a short-reach full-field coherent receiver. While ADC power dissipation scales linearly with sampling rate but exponentially with resolution [12], CPR power dissipation scales linearly with bit rate but quadratically with modulation format. As we increase the modulation format, the resolution needs to be increased, which further increases CPR power dissipation.

Fig. 3 shows a CPR design [13] based on the blind phase search (BPS) algorithm. Here we can discern estimation blocks and a phase compensation block. While the compensation has to be done in many parallel lanes to meet the required total CPR bit rate, the estimation is only partly parallelized. Rather, as much as possible of the estimation, especially the later portions of the algorithm, is handled without parallelization to reduce circuit area and power dissipation.

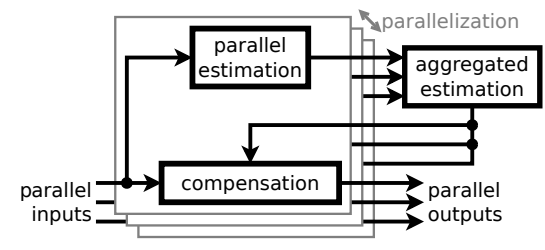

Fig. 3. CPR block diagram.

Several proposed CPR algorithms use multiple estimationcompensation stages to improve performance. But in a 32-GBd 16QAM CPR module, with an energy efficiency of $1.1 \mathrm{pJ} / \mathrm{bit}$ [13], 25\% is dissipated in the compensation block. Thus, cascading of up to four separate estimation-compensation stages [14] will be costly in terms of power dissipation. In contrast, the use of pilot symbols makes CPR implementations less complex. Here, estimation is straightforward to implement and, thus, the compensation block dominates the total power dissipation of a pilot-based CPR module, whose energy efficiency is $0.38 \mathrm{pJ} / \mathrm{bit}$ for a 32-GBd 16-QAM design [13].

Simulation run-time is not only an issue for FEC, but also for deep-BER analysis of DSP. For example, to analyze a CPR module's sensitivity to cycle slips, the module needs to be analyzed at a hardware-centric level, to ensure the detailed logic circuit behavior is captured. One way to describe the module in such a bit-equivalent manner is to write HDL code and run this as an emulation prototype in an FPGA system. The simulation time speed-up when using one FPGA, instead of using MATLAB-HDL co-simulation on a capable workstation, was shown to be five orders of magnitude [15].

\section{CONCLUSION}

The design of power-efficient digital ASICs is essential to optical communication systems. Coherent schemes offer many advantages, but they are known to lead to complex DSP and FEC circuits. While DSP circuits operate on all data samples, the correction of an error is a rare operation in FEC circuits. This distinction between principles of operation, which is still to be addressed in algorithms published in the open literature, allows us to suggest two different approaches to power-efficient implementation of DSP and FEC algorithms.

\section{REFERENCES}

[1] D. A. Morero et al., "Design tradeoffs and challenges in practical coherent optical transceiver implementations," IEEE J. Lightw. Technol., vol. 34, no. 1, pp. 121-136, Jan. 2016.

[2] P. Larsson-Edefors et al., "Implementation challenges for energyefficient error correction in optical communication systems [invited]," in OSA Advanced Photonics Congress, SPPCom, July 2018, p. SpTh4F.2.

[3] C. Fougstedt et al., "Energy-efficient high-throughput VLSI architectures for product-like codes," IEEE J. Lightw. Technol., vol. 37, no. 2, pp. 477-485, Jan. 2019.

[4] K. Cushon et al., "Low-power 400-Gbps soft-decision LDPC FEC for optical transport networks," IEEE J. Lightw. Technol., vol. 34, no. 18, pp. 4304-4311, Sept. 2016.

[5] Y. Cai et al., "FPGA investigation on error-floor performance of a concatenated staircase and Hamming code for 400G-ZR forward error correction," in Opt. Fiber Commun. Conf. (OFC), Mar. 2018, p. Th4C.2.

[6] K. Cushon et al., "A high-throughput low-power soft bit-flipping LDPC decoder in 28 nm FD-SOI," in European Solid State Circuits Conf. (ESSCIRC), Sept. 2018, pp. 102-105.

[7] T. Kupfer et al., "Optimizing power consumption of a coherent DSP for metro and data center interconnects," in Opt. Fiber Commun. Conf. $(O F C)$, Mar. 2017, p. Th3G.2.

[8] C. Fougstedt et al., "ASIC design exploration for DSP and FEC of 400-Gbit/s coherent data-center interconnect receivers," in Opt. Fiber Commun. Conf. (OFC), Mar. 2020, p. Th2A.38.

[9] C. Fougstedt et al., "Dynamic equalizer power dissipation optimization," in Opt. Fiber Commun. Conf. (OFC), Mar. 2016, p. W4A.2.

[10] X. Zhou et al., "Beyond $1 \mathrm{~Tb} / \mathrm{s}$ intra-data center interconnect technology: IM-DD or coherent?” IEEE J. Lightw. Technol., vol. 38, no. 2, pp. 475484, Jan. 2020.

[11] L. Lundberg et al., "Power consumption of a minimal-DSP coherent link with a polarization multiplexed pilot-tone," in Eur. Conf. Opt. Commun. (ECOC), Sept. 2016, pp. 1190-1192.

[12] T. Drenski et al., "ADC/DAC and ASIC technology trends," in OptoElectronics and Communications Conf. (OECC), July 2019, p. TuB2-1.

[13] E. Börjeson et al., "VLSI implementations of carrier phase recovery algorithms for M-QAM fiber-optic systems," IEEE J. Lightw. Technol., 2020, Early access, doi: 10.1109/JLT.2020.2976166.

[14] S. M. Bilal et al., "Multistage carrier phase estimation algorithms for phase noise mitigation in 64-quadrature amplitude modulation optical systems," IEEE J. Lightw. Technol., vol. 32, no. 17, pp. 2973-2980, Sept. 2014.

[15] E. Börjeson et al., "Towards FPGA emulation of fiber-optic channels for deep-BER evaluation of DSP implementations [invited]," in OSA Advanced Photonics Congress, SPPCom, July 2019, p. SpTh1E.4. 